\title{
PATTERNS IN ODD KHOVANOV HOMOLOGY
}

\author{
ALEXANDER SHUMAKOVITCH
}

\begin{abstract}
We investigate properties of the odd Khovanov homology, compare and contrast them with those of the original (even) Khovanov homology, and discuss applications of the odd Khovanov homology to other areas of knot theory and low-dimensional topology. We show that it provides an effective upper bound on the Thurston-Bennequin number of Legendrian links and can be used to detect quasi-alternating knots. A potential application to detecting transversely non-simple knots is also mentioned.
\end{abstract}

\section{INTRODUCTION}

Khovanov homology is a special case of categorification, a novel approach to construction of knot (or link) invariants that is being actively developed over the last decade after a seminal paper Kh1] by Mikhail Khovanov. The idea of categorification is to replace a known polynomial knot (or link) invariant with a family of chain complexes, such that the coefficients of the original polynomial are the Euler characteristics of these complexes. Although the chain complexes themselves depend heavily on a diagram that represents the link, their homology depend solely on the isotopy class of the link. Khovanov homology categorifies the Jones polynomial [J].

More specifically, let $L$ be an oriented link in $\mathbb{R}^{3}$ represented by a planar diagram $D$ and let $J_{L}(q)$ be a version of the Jones polynomial of $L$ that satisfies the following identities (called the Jones skein relation and normalization):

$$
-q^{-2} J_{+}(q)+q^{2} J_{\AA}(q)=(q-1 / q) J_{0}(q) ; \quad J \bigcirc(q)=q+1 / q .
$$

The skein relation should be understood as relating the Jones polynomials of three links whose planar diagrams are identical everywhere except in a small disk, where they are different as depicted in (1.1). The normalization fixes the value of the Jones polynomial on the trivial knot. $J_{L}(q)$ is a Laurent polynomial in $q$ for every link $L$ and is completely determined by its skein relation and normalization. In our paper we also make use of another version of the Jones polynomial, denoted $\widetilde{J}_{L}(q)$, that satisfies the same skein relation 11.1 but is normalized to equal 1 on the trivial knot.

In [Kh1] Mikhail Khovanov assigned to $D$ a family of Abelian groups $\mathcal{H}^{i, j}(L)$ whose isomorphism classes depend on the isotopy class of $L$ only. These groups are defined as homology groups of an appropriate (graded) chain complex $\mathcal{C}^{i, j}(D)$ with integer coefficients. Groups $\mathcal{H}^{i, j}(L)$ are nontrivial for finitely many values of the pair $(i, j)$ only. The gist of the categorification is that the graded Euler

The author was partially supported by NSF grant DMS-0707526. 
characteristic of the Khovanov chain complex equals $J_{L}(q)$ :

$$
J_{L}(q)=\sum_{i, j}(-1)^{i} q^{j} h^{i, j}(L)
$$

where $h^{i, j}(L)=\operatorname{rk}\left(\mathcal{H}^{i, j}(L)\right)$, the Betti numbers of $\mathcal{H}$. The reader is referred to [BN1, Kh1] for detailed treatment.

In 2007, Ozsváth, Rasmussen and Szabó introduced ORS an odd version of the Khovanov homology. The odd Khovanov homology equals the original (even) one modulo 2 and, in particular, categorifies the same Jones polynomial. On the other hand, the odd and even homology theories often have drastically different properties (see Sections 2 and 3 for details). The odd Khovanov homology appears to be one of the connecting links between Khovanov and Heegaard-Floer homology theories OS2.

In this paper, we investigate properties of the odd Khovanov homology and compare them to those of the even one. We also look at several applications of the even and odd Khovanov homology theories, such as giving upper bounds on the Thurston-Bennequin number of Legendrian links and detecting quasi-alternating links, and observe that the odd homology often gives a stronger result than the even one. Most of the experimental results that are referred to in the paper were obtained with KhoHo, a program by the author for computing and studying Khovanov homology Sh1.

This paper is organized as follows. In Section 2 we give a definition of the odd Khovanov homology. Our exposition is self-contained, but the reader is assumed to be familiar with the original Khovanov construction. We compare even and odd Khovanov homology theories with each other and list their basic properties in Section 3. Section 4 is devoted to some of the more important applications of the odd Khovanov homology to other areas of low-dimensional topology.

The author is grateful to Mikhail Khovanov and Lenhard Ng for many fruitful discussions during the work on this paper. He is also thankful to the referee for several helpful comments and suggestions on the draft version of this paper.

\section{Definition of the odd Khovanov homology}

In this section we give a brief outline of the odd Khovanov homology theory following [RS]. Our setting is slightly more general than the one in the Introduction as we allow different coefficient rings, not only $\mathbb{Z}$.

2.1. Algebraic preliminaries. Let $R$ be a commutative ring with unity. In this paper, we are mainly interested in the cases when $R=\mathbb{Z}, \mathbb{Q}$, or $\mathbb{Z}_{2}$. If $M$ is a graded $R$-module, we denote its homogeneous component of degree $j$ by $M_{j}$. For an integer $k$, the shifted module $M\{k\}$ is defined as having homogeneous components $M\{k\}_{j}=M_{j-k}$. In the case when $M$ is free and finite dimensional, we define its graded dimension as the power series $\operatorname{dim}_{q}(M)=\sum_{j \in \mathbb{Z}} q^{j} \operatorname{dim}\left(M_{j}\right)$ in variable $q$.

Finally, if $(\mathcal{C}, d)=\cdots \longrightarrow \mathcal{C}^{i-1} \stackrel{d^{i-1}}{\longrightarrow} \mathcal{C}^{i} \stackrel{d^{i}}{\longrightarrow} \mathcal{C}^{i+1} \longrightarrow \cdots$ is a (co)chain complex of graded free $R$-modules such that all differentials $d^{i}$ are graded of degree 0 with respect to the internal grading, we define its graded Euler characteristic as $\chi_{q}(\mathcal{C})=$ $\sum_{i \in \mathbb{Z}}(-1)^{i} \operatorname{dim}_{q}\left(\mathcal{C}^{i}\right)$. 

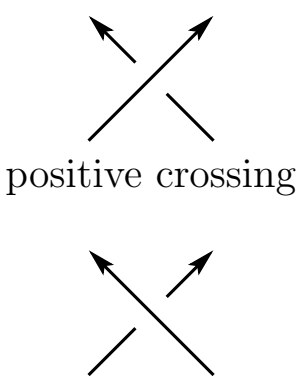

negative crossing

Figure 1. Positive and negative crossings

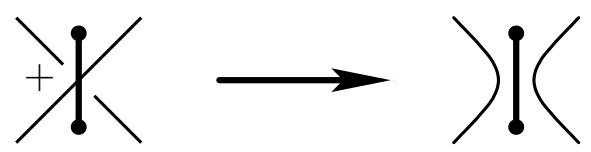

positive marker

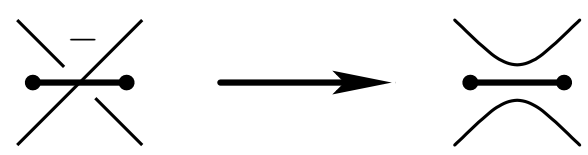

negative marker
Figure 2. Positive and negative markers and the corresponding resolutions of a diagram.

Remark. One can think of a (co)chain complex of graded $R$-modules as a bigraded $R$-module where the homogeneous components are indexed by pairs of numbers $(i, j) \in \mathbb{Z}^{2}$. Under this point of view, the differentials are graded of bidegree $(1,0)$.

2.2. Odd Khovanov chain complex. Let $L$ be an oriented link and $D$ its planar diagram. We assign a number \pm 1 , called sign, to every crossing of $D$ according to the rule depicted in Figure 1 . The sum of these signs over all the crossings of $D$ is called the writhe number of $D$ and is denoted by $w(D)$.

Every crossing of $D$ can be resolved in two different ways according to a choice of a marker, which can be either positive or negative, at this crossing (see Figure 2). A collection of markers chosen at every crossing of a diagram $D$ is called a (Kauffman) state of $D$. For a diagram with $n$ crossings, there are, obviously, $2^{n}$ different states. Denote by $\sigma(s)$ the the number of positive markers minus the number of negative ones in a given state $s$. Define

$$
i(s)=\frac{w(D)-\sigma(s)}{2}, \quad j(s)=\frac{3 w(D)-\sigma(s)}{2} .
$$

Since both $w(D)$ and $\sigma(s)$ are congruent to $n$ modulo $2, i(s)$ and $j(s)$ are always integers. For a given state $s$, the result of the resolution of $D$ at each crossing according to $s$ is a family $D_{s}$ of disjointly embedded circles. Denote the number of these circles by $\left|D_{s}\right|$.

For each state $s$ of $D$, we assign a free graded $R$-module $\Lambda(s)$ as follows. Label all circles from the resolution $D_{s}$ by some independent variables, say, $X_{1}^{s}, X_{2}^{s}, \ldots, X_{\left|D_{s}\right|}^{s}$ and let $V_{s}=V\left(X_{1}^{s}, X_{2}^{s}, \ldots, X_{\left|D_{s}\right|}^{s}\right)$ be a free $R$-module generated by them. We define $\Lambda(s)=\Lambda^{*}\left(V_{s}\right)$, the exterior algebra of $V_{s}$. Then $\Lambda(s)=\Lambda^{0}\left(V_{s}\right) \oplus \Lambda^{1}\left(V_{s}\right) \oplus \cdots \oplus$ $\Lambda^{\left|D_{s}\right|}\left(V_{s}\right)$ and we grade $\Lambda(s)$ by specifying $\Lambda(s)_{\left|D_{s}\right|-2 k}=\Lambda^{k}\left(V_{s}\right)$ for each $0 \leq k \leq$ $\left|D_{s}\right|$, where $\Lambda(s)_{\left|D_{s}\right|-2 k}$ is the homogeneous component of $\Lambda(s)$ of degree $\left|D_{s}\right|-2 k$. It is an easy exercise for the reader to check that $\operatorname{dim}_{q}(\Lambda(s))=\left(q+q^{-1}\right)^{\left|D_{s}\right|}$.

Let $\mathcal{C}_{\text {odd }}^{i}(D)=\bigoplus_{i(s)=i} \Lambda(s)\{j(s)\}$ for each $i \in \mathbb{Z}$. In order to arrange these modules into a graded chain complex $\mathcal{C}_{\text {odd }}(D)$, we need to define a (graded) differential $d_{\text {odd }}^{i}: \mathcal{C}_{\text {odd }}^{i}(D) \rightarrow \mathcal{C}_{\text {odd }}^{i+1}(D)$ of degree 0 . But even before the chain complex structure on $\mathcal{C}_{\text {odd }}(D)$ is defined, its (graded) Euler characteristic makes sense. Similarly to the case of the even Khovanov homology, one can easily verify that $\chi_{q}\left(\mathcal{C}_{\text {odd }}(D)\right)=J_{L}(q)$. In fact, $\mathcal{C}_{\text {odd }}(D) \simeq \mathcal{C}(D)$ as bigraded $R$-modules ORS. 


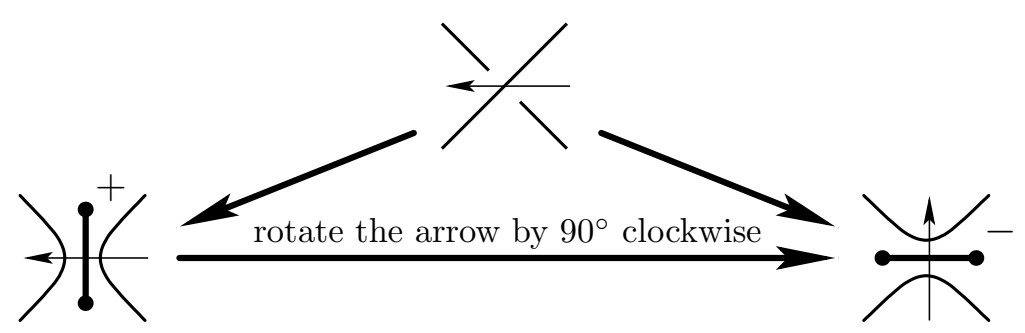

Figure 3. Choice of arrows at the diagram crossings

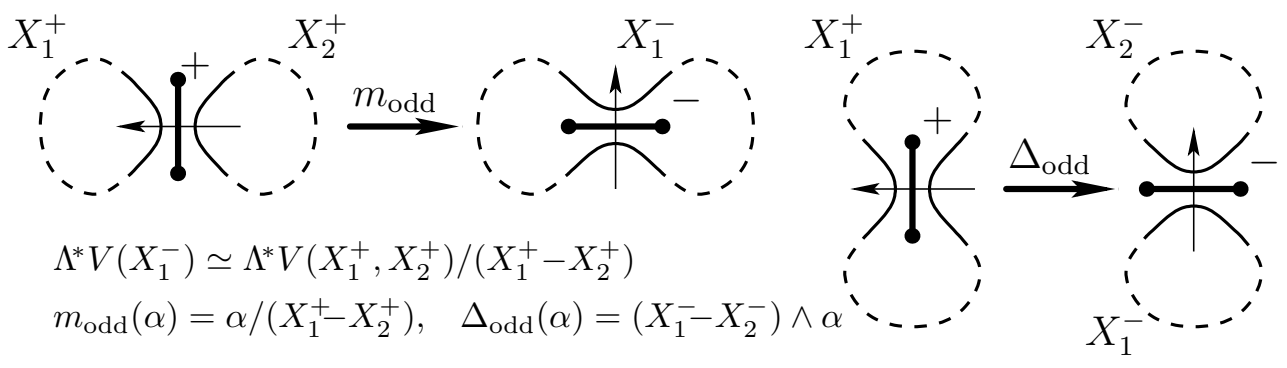

FIgURE 4. Diagram resolutions corresponding to adjacent states and maps between the free $R$-modules assigned to these resolutions

To define a differential on $\mathcal{C}_{\text {odd }}$, we introduce an additional structure, a choice of an arrow at each crossing of $D$ that is parallel to the negative marker at that crossing (see Figure 3). There are obviously $2^{n}$ such choices. For every state $s$ on $D$, we place arrows that connect two branches of $D_{s}$ near each (former) crossing according to the rule from Figure 3

Let $s_{+}$and $s_{-}$be two states of $D$ that differ at a single crossing, where $s_{+}$has a positive marker while $s_{-}$has a negative one. We call two such states adjacent. In this case, $\sigma\left(s_{-}\right)=\sigma\left(s_{+}\right)-2$ and, consequently, $i\left(s_{-}\right)=i\left(s_{+}\right)+1$ and $j\left(s_{-}\right)=$ $j\left(s_{+}\right)+1$. Consider now the resolutions of $D$ corresponding to $s_{+}$and $s_{-}$. One can readily see that $D_{s_{-}}$is obtained from $D_{s_{+}}$by either merging two circles into one or splitting one circle into two (see Figure 4). All the circles that do not pass through the crossing at which $s_{+}$and $s_{-}$differ, remain unchanged. We define $d_{s_{+}: s_{-}}: \Lambda\left(s_{+}\right) \rightarrow \Lambda\left(s_{-}\right)$as either $m_{\text {odd }}$ or $\Delta_{\text {odd }}$ depending on whether the circles merge or split, where $m_{\text {odd }}$ and $\Delta_{\text {odd }}$ are as follows.

If $s_{-}$is obtained from $s_{+}$by merging two circles together, then we have that $\Lambda\left(s_{-}\right) \simeq \Lambda\left(s_{+}\right) /\left(X_{1}^{+}-X_{2}^{+}\right)$, where $X_{1}^{+}$and $X_{2}^{+}$are the generators of $V_{s_{+}}$corresponding to the two merging circles, as depicted in Figure 4 . We define $m_{\text {odd }}$ : $\Lambda\left(s_{+}\right) \rightarrow \Lambda\left(s_{-}\right)$to be this isomorphism composed with the projection $\Lambda\left(s_{+}\right) \rightarrow$ $\Lambda\left(s_{+}\right) /\left(X_{1}^{+}-X_{2}^{+}\right)$.

The case when one circle splits into two is more interesting. Let $X_{1}^{-}$and $X_{2}^{-}$be the generators of $V_{s_{-}}$corresponding to these two circles such that the arrow points from $X_{1}^{-}$to $X_{2}^{-}$(see Figure 44). Now for each generator $X_{k}^{+}$of $V_{s_{+}}$, we define $\Delta_{\text {odd }}\left(X_{k}^{+}\right)=\left(X_{1}^{-}-X_{2}^{-}\right) \wedge X_{\eta(k)}^{-}$where $\eta$ is the correspondence between circles in $D_{s_{+}}$and $D_{s_{-}}$. While $\eta(1)$ can equal either 1 or 2 , this choice does not affect $\Delta_{\text {odd }}\left(X_{1}^{+}\right)$since $\left(X_{1}^{-}-X_{2}^{-}\right) \wedge X_{2}^{-}=X_{1}^{-} \wedge X_{2}^{-}=-X_{2}^{-} \wedge X_{1}^{-}=\left(X_{1}^{-}-X_{2}^{-}\right) \wedge X_{1}^{-}$. 


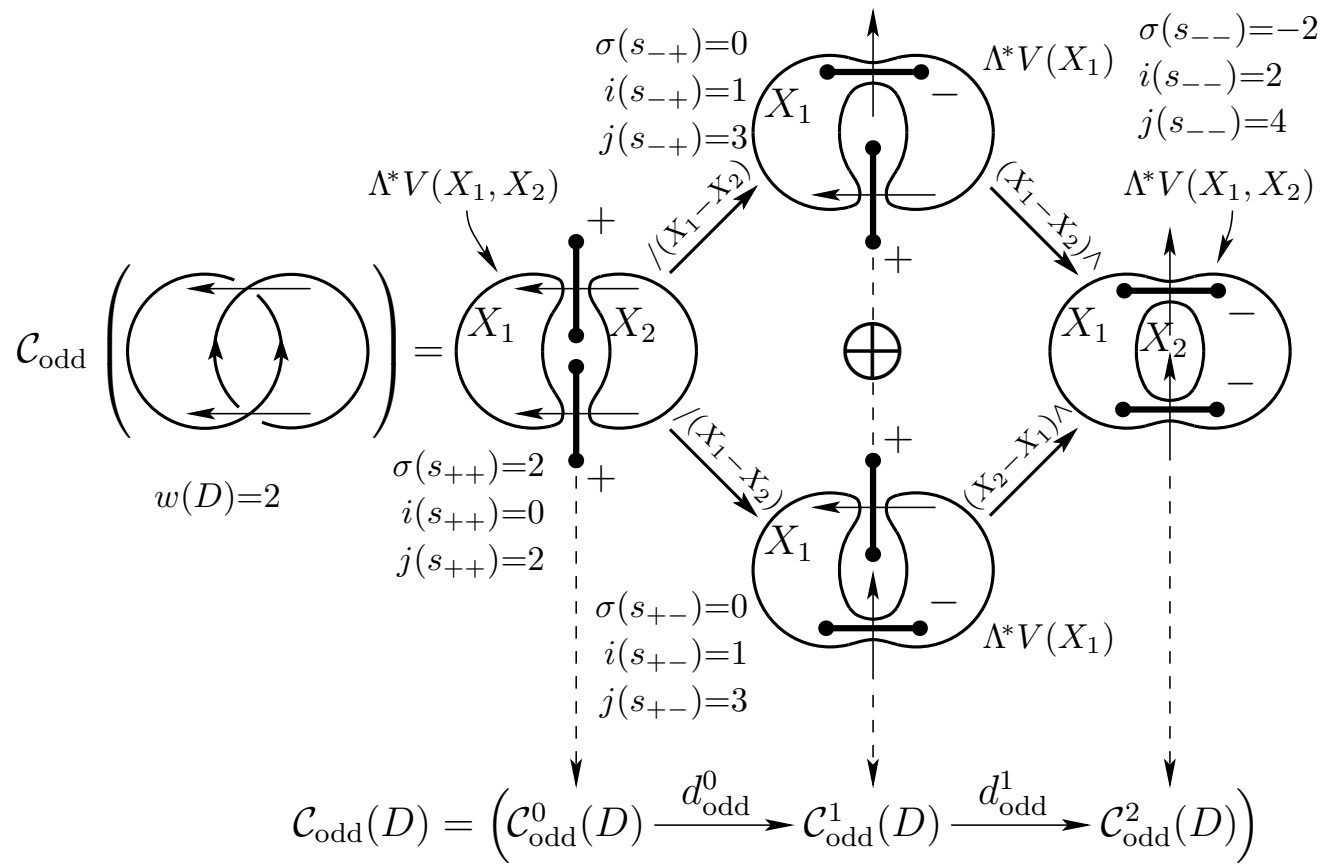

Figure 5. Odd Khovanov chain complex for the Hopf link

We need one more ingredient in order to finish the definition of the differential on $\mathcal{C}_{\text {odd }}(D)$. Namely, to each adjacent pair of states $\left(s_{+}, s_{-}\right)$, we assign a sign $\varepsilon\left(s_{+}, s_{-}\right) \in\{ \pm 1\}$. This choice of a sign for each adjacent pair is called an edge assignment. The etymology of this term will become clear in a few paragraphs. Finally, let $d_{\text {odd }}^{i}=\sum_{\left(s_{+}, s_{-}\right)} \varepsilon\left(s_{+}, s_{-}\right) d_{s_{+}: s_{-}}$, where $\left(s_{+}, s_{-}\right)$runs over all adjacent pairs of states with $i\left(s_{+}\right)=i$. Our goal is to choose an edge assignment in such a way that $d_{\text {odd }}: \mathcal{C}(D) \rightarrow \mathcal{C}(D)$ is indeed a differential, that is, $d_{\text {odd }}^{i+1} \circ d_{\text {odd }}^{i}=0$ for every $i$. The following Theorem guarantees us that it is always possible.

2.2.A. Theorem (Ozsváth-Rasmussen-Szabó ORS). It is possible to assign a $\operatorname{sign} \varepsilon\left(s_{+}, s_{-}\right)$to each adjacent pair of states $\left(s_{+}, s_{-}\right)$in such a way that $\mathcal{C}_{\text {odd }}(D ; R)$ equipped with the differential $d_{\mathrm{odd}}$ defined above becomes a graded (co)chain complex. The homology $\mathcal{H}_{\text {odd }}(L ; R)$ of $\mathcal{C}_{\text {odd }}(D ; R)$ does not depend on the choice of arrows at the crossings, the choice of edge assignment, and some other choices needed in the construction. Moreover, the isomorphism class of $\mathcal{H}_{\mathrm{odd}}(L ; R)$ is a link invariant that categorifies $J_{L}(q)$, a version of the Jones polynomial defined by (1.1).

2.2.B. Definition ( $\mathrm{ORS}]$ ). $\mathcal{H}_{\text {odd }}(L ; R)$ is called the odd Khovanov homology of $L$ over a ring of coefficients $R$. If $R$ is omitted from the notation, integer coefficients are assumed.

2.2.C. By comparing the definitions of $\mathcal{C}_{\text {odd }}\left(D ; \mathbb{Z}_{2}\right)$ and $\mathcal{C}\left(D ; \mathbb{Z}_{2}\right)$, it is easy to see that they are isomorphic as graded chain complexes (since the signs do not matter modulo 2$)$. It follows that $\mathcal{H}_{\text {odd }}\left(D ; \mathbb{Z}_{2}\right) \simeq \mathcal{H}\left(D ; \mathbb{Z}_{2}\right)$ as well.

2.2.D. Example. Figure 5 shows the odd Khovanov chain complex for the Hopf link with the indicated orientation. The diagram has two positive crossings, so its 
writhe number is 2 . Let $s_{ \pm \pm}$be the four possible resolutions of this diagram, where each "+" or "-" describes the sign of the marker at the corresponding crossing. By looking at Figure 5, one easily determines that

$$
\begin{aligned}
& \mathcal{C}_{\text {odd }}^{0}(D)=\Lambda\left(s_{++}\right)\{2\}=\Lambda^{*} V\left(X_{1}, X_{2}\right)\{2\} ; \\
& \mathcal{C}_{\text {odd }}^{1}(D)=\Lambda\left(s_{+-}\right)\{3\} \oplus \Lambda\left(s_{-+}\right)\{3\}=\left(\Lambda^{*} V\left(X_{1}\right) \oplus \Lambda^{*} V\left(X_{1}\right)\right)\{3\} ; \\
& \mathcal{C}_{\text {odd }}^{2}(D)=\Lambda\left(s_{--}\right)\{4\}=\Lambda^{*} V\left(X_{1}, X_{2}\right)\{4\} .
\end{aligned}
$$

It is convenient to arrange the four resolutions in the corners of a square placed in the plane in such a way that its diagonal from $s_{++}$to $s_{--}$is horizontal. Then the edges of this square correspond to the maps between the adjacent states (see Figure 5).

In general, $2^{n}$ resolutions of a diagram $D$ with $n$ crossings can be arranged into an $n$-dimensional cube of resolutions, where vertices correspond to the $2^{n}$ states of $D$. The edges of this cube connect adjacent pairs of states and can be oriented from $s_{+}$to $s_{-}$. Every edge is assigned either $m_{\text {odd }}$ or $\Delta_{\text {odd }}$ with the sign $\varepsilon\left(s_{+}, s_{-}\right)$, as described above. The differential $d_{\text {odd }}^{i}$ restricted to each summand $\Lambda(s)$ with $i(s)=i$ equals the sum of all the maps assigned to the edges that originate at $s$.

Remark. If one forgets about the signs on the edges, then each square (that is, a 2-dimensional face) in the cube of resolutions is either commutative, or anticommutative, or both. The latter case means that both double-composites corresponding to the square are trivial. This is a major departure from the situation in the even case, where each square is commutative Kh1. The role of the signs $\varepsilon\left(s_{+}, s_{-}\right)$is to make all of the squares anti-commutative.

Remark. In the example 2.2.D the only square of resolutions is anti-commutative, so no adjustment of signs is needed.

Remark. There is no explicit construction for finding edge assignments for the cube of resolutions in the case of the odd Khovanov chain complex. Theorem 2.2.A only ensures that signs exist. On the other hand, such an assignment is fairly straightforward for the even Khovanov chain complex.

2.3. Reduced odd Khovanov homology. Similar to the even case [Kh2, there is a reduced version of the odd Khovanov homology:

2.3.A. TheOREM ( $\mathrm{ORS})$. For every link $L$, there exists a bigraded abelian group $\widetilde{\mathcal{H}}_{\text {odd }}(L ; R)$ such that $\mathcal{H}_{\text {odd }}(L ; R) \simeq \widetilde{\mathcal{H}}_{\text {odd }}(L ; R)\{1\} \oplus \widetilde{\mathcal{H}}_{\text {odd }}(L ; R)\{-1\}$.

$\widetilde{\mathcal{H}}_{\text {odd }}(L ; R)$ is called the reduced odd Khovanov homology of a link $L$ over $R$. Theorem 2.3.A implies that the reduced and non-reduced odd homology determine each other completely. It is therefore enough to consider the reduced version of the odd Khovanov homology only. This contrasts with the even case where there are several examples of pairs of knots that have the same rational Khovanov homology, but different rational reduced ones.

Remark. One of the main differences between the even and odd reduced Khovanov homologies is that the former depends on the choice of a link component, while the latter does not. This difference disappears over $\mathbb{Z}_{2}$.

2.3.B. Theorem $(\underline{\operatorname{Sh} 2})$. $\mathcal{H}\left(L ; \mathbb{Z}_{2}\right) \simeq \widetilde{\mathcal{H}}\left(L ; \mathbb{Z}_{2}\right) \otimes_{\mathbb{Z}_{2}} A_{\mathbb{Z}_{2}}$ for every link $L$, where $A_{\mathbb{Z}_{2}}=\mathbb{Z}_{2}[X] / X^{2}$ is the algebra of truncated polynomials graded by $\operatorname{deg}(1)=1$ 
and $\operatorname{deg}(X)=-1$. In particular, $\widetilde{\mathcal{H}}\left(L ; \mathbb{Z}_{2}\right)$ does not depend on the choice of a component of $L$.

The odd Khovanov homology should provide an insight into interrelations between Khovanov and Heegaard-Floer OS1 homology theories. Its definition was motivated by the following result.

2.3.C. TheOrem (Ozsváth-Szabó OS2]). For each link L with a diagram $D$, there exists a spectral sequence with $E^{1}=\widetilde{\mathcal{C}}\left(D ; \mathbb{Z}_{2}\right)$ and $E^{2}=\widetilde{\mathcal{H}}\left(L ; \mathbb{Z}_{2}\right)$ that converges to the $\mathbb{Z}_{2}$-Heegaard-Floer homology $\widehat{H F}\left(\Sigma(L) ; \mathbb{Z}_{2}\right)$ of the double branched cover $\Sigma(L)$ of $S^{3}$ along $L$.

2.3.D. CONJECTURE. There exists a spectral sequence that starts with $\widetilde{\mathcal{C}}_{\text {odd }}(D ; \mathbb{Z})$ and $\widetilde{\mathcal{H}}_{\text {odd }}(L ; \mathbb{Z})$ and converges to $\widehat{H F}(\Sigma(L) ; \mathbb{Z})$.

\section{Comparison Between even And odd Khovanov homology}

In this section we summarize the main differences and similarities in properties exhibited by the even and odd versions of Khovanov homology and list related constructions.

3.A. Let $L$ be an oriented link and $D$ its planar diagram. Then

- $\mathcal{H}_{\text {odd }}\left(L ; \mathbb{Z}_{2}\right) \simeq \mathcal{H}\left(L ; \mathbb{Z}_{2}\right)$ and $\widetilde{\mathcal{H}}_{\text {odd }}\left(L ; \mathbb{Z}_{2}\right) \simeq \widetilde{\mathcal{H}}\left(L ; \mathbb{Z}_{2}\right)$.

- $\chi_{q}(\mathcal{H}(L ; R))=\chi_{q}\left(\mathcal{H}_{\text {odd }}(L ; R)\right)=J_{L}(q)$ and $\chi_{q}(\widetilde{\mathcal{H}}(L ; R))=\chi_{q}\left(\widetilde{\mathcal{H}}_{\text {odd }}(L ; R)\right)=\widetilde{J}_{L}(q)$.

- $\mathcal{H}\left(L ; \mathbb{Z}_{2}\right) \simeq \widetilde{\mathcal{H}}\left(L ; \mathbb{Z}_{2}\right) \otimes_{\mathbb{Z}_{2}} A_{\mathbb{Z}_{2}}$ Sh2 and $\mathcal{H}_{\text {odd }}(L ; R) \simeq \widetilde{\mathcal{H}}_{\text {odd }}(L ; R)\{1\} \oplus$ $\widetilde{\mathcal{H}}_{\text {odd }}(L ; R)\{-1\}$ ORS. On the other hand, $\mathcal{H}(L ; \mathbb{Z})$ and $\mathcal{H}(L ; \mathbb{Q})$ do not split in general.

- For links, $\widetilde{\mathcal{H}}\left(L ; \mathbb{Z}_{2}\right)$ and $\widetilde{\mathcal{H}}_{\text {odd }}(L ; R)$ do not depend on the choice of a component on which a base point lies. This is, in general, not the case for $\widetilde{\mathcal{H}}(L ; \mathbb{Z})$ and $\widetilde{\mathcal{H}}(L ; \mathbb{Q})$.

- If $L$ is a non-split alternating link, then $\mathcal{H}(L ; \mathbb{Q}), \widetilde{\mathcal{H}}(L ; R)$, and $\widetilde{\mathcal{H}}_{\text {odd }}(L ; R)$ are completely determined by the Jones polynomial and signature of $L[\mathrm{Kh} 2$, L, ORS.

- $\mathcal{H}\left(L ; \mathbb{Z}_{2}\right)$ and $\mathcal{H}_{\text {odd }}(L ; \mathbb{Z})$ are invariant under link mutations $[\mathrm{B}, \mathrm{W} 2$. On the other hand, $\mathcal{H}(L ; \mathbb{Z})$ is known not to be preserved under a mutation that exchanges components of a link W1 and under a cabled (or genus 2) mutation DGShT. It is unclear whether $\mathcal{H}(L ; \mathbb{Z})$ is invariant under a component-preserving mutation.

- $\mathcal{H}(L ; \mathbb{Z})$ almost always has torsion (except for several special cases), but usually of order 2 . The first knot with 4-torsion is the $(4,5)$-torus knot that has 15 crossings. The first known knot with 3 -torsion is the $(5,6)$ torus knot with 24 crossings. On the other hand, $\mathcal{H}_{\text {odd }}(L ; \mathbb{Z})$ was observed to have torsion of various orders even for knots with relatively few crossings (see remark on page 15), although orders 2 and 3 are the most popular.

- $\widetilde{\mathcal{H}}(L ; \mathbb{Z})$ has very little torsion. The first knot with torsion has 13 crossings. On the other hand, $\widetilde{\mathcal{H}}_{\text {odd }}(L ; \mathbb{Z})$ has as much torsion as $\mathcal{H}_{\text {odd }}(L ; \mathbb{Z})$ (see Theorem 2.3.A.

Remark. The properties above show that $\widetilde{\mathcal{H}}_{\text {odd }}(L ; \mathbb{Z})$ behaves similarly to $\widetilde{\mathcal{H}}\left(L ; \mathbb{Z}_{2}\right)$ but not to $\widetilde{\mathcal{H}}(L ; \mathbb{Z})$. This is one of its main features (see 2.3.C and 2.3.D. 
3.1. Distinguishing knots. The odd Khovanov homology appears to be somewhat stronger of a knot invariant than the even one, although independent. Among 313230 prime knots with up to 15 crossings (111528 alternating and 201702 nonalternating), there are 4377 pairs of knots (8754 if counted with mirror images, as none of these knots are amphicheiral) that have the same even Khovanov homology but different odd ones. The first knots that are distinguished by the odd homology but not the even one are $11_{1}^{n}$ and $\overline{12}_{577}^{n}$. On the other hand, there are only 107 (214 if counted with mirror images) pairs of prime knots with up to 15 crossings that have the same odd Khovanov homology but different even ones. The first knots distinguished by the even homology but not the odd one are $13_{2640}^{n}$ and $15_{124915}^{n}$. We don't know any special properties of these knots.

Remark. Throughout this paper we use the following notation for knots: knots with 10 crossings or less are numbered according to Rolfsen's knot table [Ro] and knots with 11 crossings or more are numbered according to the knot table from Knotscape [HTh. Mirror images of knots from either table are denoted with a bar on top. For example, $\overline{9}_{46}$ is the mirror image of the knot number 46 with 9 crossings from Rolfsen's table and $16_{197566}^{n}$ is the non-alternating knot number 197566 with 16 crossings from Knotscape's table.

It is interesting to notice that the odd Khovanov homology is the same for all almost mutant knots DGShT with up to 16 crossings. There is currently no explanation for this fact. Even homology, on the other hand, is known to distinguish some of them.

\subsection{Homological thickness.}

3.2.A. Definition. Let $L$ be a link. The homological width of $L$ over a ring $R$ is the minimal number of adjacent diagonals $j-2 i=$ const such that $\mathcal{H}(L ; R)$ is zero outside of these diagonals. It is denoted by $\mathrm{hw}_{R}(L)$. The reduced homological width, $\widetilde{\mathrm{hw}}_{R}(L)$ of $L$, odd homological width, $\operatorname{ohw}_{R}(L)$ of $L$, and reduced odd homological width, $\widetilde{\mathrm{ohw}}_{R}(L)$ of $L$ are defined similarly.

3.2.B. It follows from 2.3.A that $\widetilde{\mathrm{ohw}}_{R}(L)=\mathrm{ohw}_{R}(L)-1$. The same holds true in the case of the even Khovanov homology over $\mathbb{Q}: \widetilde{h w}_{\mathbb{Q}}(L)=\operatorname{hw}_{\mathbb{Q}}(L)-1$ (see [Kh2]).

3.2.C. Definition. A link $L$ is said to be homologically thin over a ring $R$, or simply $R$ H-thin, if $\operatorname{hw}_{R}(L)=2$. $L$ is homologically thick, or $R$ H-thick, otherwise. We define odd-homologically thin and thick, or simply $R \mathrm{OH}$-thin and $R \mathrm{OH}$-thick, links similarly.

3.2.D. Theorem (Lee, Ozsváth-Rasmussen-Szabó, Manolescu-Ozsváth L, ORS, MO]). Quasi-alternating links (see Section 4.2 for the definition) are RH-thin and ROH-thin for every ring $R$. In particular, this is true for non-split alternating links.

3.2.E. TheOREm (Khovanov $[\mathrm{Kh} 2$ ). Adequate links are $R H$-thick for every $R$.

3.2.F. Homological thickness of a link $L$ often does not depend on the base ring. The first prime knot with $\mathrm{hw}_{\mathbb{Q}}(L)<\operatorname{hw}_{\mathbb{Z}_{2}}(L)$ and $\mathrm{hw}_{\mathbb{Q}}(L)<\mathrm{hw}_{\mathbb{Z}}(L)$ is $15_{41127}^{n}$ with 15 crossings (see Figure 6). The first prime knot that is $\mathbb{Q H}$-thin but $\mathbb{Z H}$ thick, $16_{197566}^{n}$, has 16 crossings (see Figure 7 ). Its mirror image, $\overline{16}_{197566}^{n}$ is both 


\begin{tabular}{|r||l|l|l|l|l|l|l|l|l|l|l||}
\hline \hline & -7 & -6 & -5 & -4 & -3 & -2 & -1 & 0 & 1 & 2 & 3 \\
\hline \hline 8 & & & & & & & & & & & $\mathbf{1}$ \\
\hline 6 & & & & & & & & & & $\mathbf{1}$ & \\
\hline 4 & & & & & & & & & $\mathbf{1}$ & & \\
\hline 2 & & & & & & & $\mathbf{1}$ & $\mathbf{2}$ & & & \\
\hline 0 & & & & & & $\mathbf{1}$ & & $\mathbf{1}$ & & & \\
\hline-2 & & & & & $\mathbf{1}$ & $\mathbf{1}$ & & & & & \\
\hline-4 & & & & $\mathbf{2}$ & $\mathbf{1}$ & & & & & & \\
\hline-6 & & & $\mathbf{1}$ & & & & & & & & \\
\hline-8 & & $\mathbf{1}$ & & & & & & & & & \\
\hline-10 & $\mathbf{1}$ & & & & & & & & & & \\
\hline \hline
\end{tabular}

$$
\mathrm{hw}_{\mathbb{Q}}=3, \quad \widetilde{\mathrm{hw}_{\mathbb{Q}}}=2, \quad \mathrm{hw}_{\mathbb{Z}}=4, \quad \widetilde{\mathrm{hw}_{\mathbb{Z}}}=3
$$

\begin{tabular}{|r||r|r|r|r|r|r|r|r|r|r|r||}
\hline \hline-7 & -6 & -5 & -4 & -3 & -2 & -1 & 0 & 1 & 2 & 3 \\
\hline \hline 8 & & & & & & & & & & & $\mathbf{1}$ \\
\hline 6 & & & & & & & & & & $\mathbf{1}$ & \\
\hline 4 & & & & & & & & & $\mathbf{1}$ & & \\
\hline 0 & & & & & & & & $\mathbf{1}, \mathbf{1}_{\mathbf{2}}$ & & & \\
\hline-2 & & & & & & $\mathbf{1} \mathbf{6}$ & & & & & \\
\hline-4 & & & & $\mathbf{1}$ & $\mathbf{1}_{\mathbf{2}}$ & & & & & & \\
\hline-6 & & & $\mathbf{1}$ & & & & & & & & \\
\hline-8 & & $\mathbf{1}$ & & & & & & & & & \\
\hline-10 & $\mathbf{1}$ & & & & & & & & & & \\
\hline \hline
\end{tabular}

$$
\operatorname{ohw}_{\mathbb{Q}}=4, \quad \widetilde{\operatorname{ohw}_{\mathbb{Q}}}=3, \quad \widetilde{o h w}_{\mathbb{Z}}=4, \quad \widetilde{\operatorname{ohw}}_{\mathbb{Z}}=3
$$

Figure 6. Integral reduced even Khovanov homology (above) and odd Khovanov Homology (below) of the knot $15_{41127}^{n}$

$\mathbb{Q H}$ - and $\mathbb{Z}$ H-thin but is $\mathbb{Z}_{2} \mathrm{H}$-thick with $\mathcal{H}^{-8,-21}\left(\overline{16}_{197566}^{n} ; \mathbb{Z}_{2}\right) \simeq \mathbb{Z}_{2}$, for example, because of the Universal Coefficient Theorem. Also observe that $\mathcal{H}^{9,25}\left(16_{197566}^{n} ; \mathbb{Z}\right)$ and $\mathcal{H}^{-8,-25}\left(\overline{16}_{197566}^{n} ; \mathbb{Z}\right)$ have 4 -torsion, shown in a small box in the tables.

Remark. Tables in Figures 6 and 7 tabulate non-zero homology groups of the corresponding knots where the $i$-grading is represented horizontally and the $j$-grading 


\begin{tabular}{|c|c|c|c|c|c|c|c|c|c|c|c|c|c|}
\hline & -2 & -1 & 0 & 1 & 2 & 3 & 4 & 5 & 6 & 7 & 8 & 99 & 10 \\
\hline 29 & & & & & & & & & & & & & 1 \\
\hline 27 & & & & & & & & & & & & 4 & $1_{2}$ \\
\hline 25 & & & & & & & & & & & 7 & $1,3_{2} 1_{4}$ & \\
\hline 23 & & & & & & & & & & 12 & $4,8_{2}$ & & \\
\hline 21 & & & & & & & & & 15 & $7,12_{2}$ & $1_{2}$ & & \\
\hline 19 & & & & & & & & 17 & $12,16_{2}$ & & & & \\
\hline 17 & & & & & & & 16 & $15,18_{2}$ & $1_{2}$ & & & & \\
\hline 15 & & & & & & 15 & $17,16_{2}$ & $1_{2}$ & & & & & \\
\hline 13 & & & & & 10 & $16,15_{2}$ & & & & & & & \\
\hline 11 & & & & 6 & $15,10_{2}$ & & & & & & & & \\
\hline 9 & & & 3 & $10,6_{2}$ & & & & & & & & & \\
\hline 7 & & 1 & $7,2_{2}$ & & & & & & & & & & \\
\hline 5 & & $2,1_{2}$ & & & & & & & & & & & \\
\hline 3 & 1 & & & & & & & & & & & & \\
\hline
\end{tabular}

The free part of $\mathcal{H}\left(16_{197566}^{n} ; \mathbb{Z}\right)$ is supported on diagonals $j-2 i=7$ and $j-2 i=9$. On the other hand, there is 2 -torsion on the diagonal $j-2 i=5$. Therefore, $16_{197566}^{n}$ is $\mathbb{Q H}$-thin, but $\mathbb{Z} \mathrm{H}$-thick and $\mathbb{Z}_{2} \mathrm{H}$-thick.

\begin{tabular}{|c|c|c|c|c|c|c|c|c|c|c|c|c|c|}
\hline & -10 & -9 & -8 & -7 & -6 & -5 & -4 & -3 & -2 & -1 & 0 & 1 & 2 \\
\hline-3 & & & & & & & & & & & & & 1 \\
\hline-5 & & & & & & & & & & & & 2 & $1_{2}$ \\
\hline-7 & & & & & & & & & & & 7 & $1,2_{2}$ & \\
\hline-9 & & & & & & & & & & 10 & $3,6_{2}$ & & \\
\hline$-11 \mid$ & & & & & & & & & 15 & $6,10_{2}$ & & & \\
\hline-13 & & & & & & & & 16 & $10,15_{2}$ & & & & \\
\hline-15 & & & & & & & $17,1_{2}$ & $15,16_{2}$ & & & & & \\
\hline-17 & & & & & & $15,1_{2}$ & $16,18_{2}$ & & & & & & \\
\hline-19 & & & & & 12 & $17,16_{2}$ & & & & & & & \\
\hline-21 & & & & $7,1_{2}$ & $15,12_{2}$ & & & & & & & & \\
\hline-23 & & & 4 & $12,8_{2}$ & & & & & & & & & \\
\hline-25 & & 1 & $7,3_{2} 1_{4}$ & & & & & & & & & & \\
\hline-27 & & $4,1_{2}$ & & & & & & & & & & & \\
\hline-29 & 1 & & & & & & & & & & & & \\
\hline
\end{tabular}

$\mathcal{H}\left(\overline{16}_{197566}^{n} ; \mathbb{Z}\right)$ is supported on diagonals $j-2 i=-7$ and $j-2 i=-9$.

But there is 2-torsion on the diagonal $j-2 i=-7$.

Therefore, $\overline{16}_{197566}^{n}$ is $\mathbb{Q H}$-thin and $\mathbb{Z} \mathrm{H}$-thin, but $\mathbb{Z}_{2} \mathrm{H}$-thick.

FiguRE 7. Integral Khovanov homology of the knots $16_{197566}^{n}$ and $\overline{16}_{197566}^{n}$ 
vertically. A table entry of $\mathbf{1}$ or $\mathbf{1}_{\mathbf{2}}$ means that the corresponding group is $\mathbb{Z}$ or $\mathbb{Z}_{2}$, respectively. In general, an entry of the form $\mathbf{a}, \mathbf{b}_{\mathbf{c}}$ would correspond to the group $\mathbb{Z}^{a} \oplus \mathbb{Z}_{c}^{b}$.

3.2.G. Odd Khovanov homology is often thicker over $\mathbb{Z}$ than the even one. This is crucial for applications (see Section 4 ). On the other hand, $\widetilde{\text { ohw }} \mathbb{Q}(L) \leq \widetilde{\mathrm{hw}_{\mathbb{Q}}}(L)$ for all but one prime knot with at most 15 crossings. The homology for this knot, $15_{41127}^{n}$, is shown in Figure 6. Please observe that $\widetilde{\mathcal{H}}_{\text {odd }}\left(15_{41127}^{n}\right)$ has 3-torsion (in gradings $(-2,-2)$ and $(-1,0))$, while $\widetilde{\mathcal{H}}\left(15_{41127}^{n}\right)$ has none.

3.3. Long exact sequence of the Khovanov homology. One of the most useful tools in studying Khovanov homology is the long exact sequence that categorifies Kauffman's unoriented skein relation for the Jones polynomial Kh1. A similar long exact sequence exists for the odd version of the homology as well ORS. If we forget about the grading, then it is clear from the construction from Section 2.2 that $\mathcal{C}_{\text {odd }}(\smile)$ is a subcomplex of $\mathcal{C}_{\text {odd }}(\nearrow)$ and $\left.\mathcal{C}_{\text {odd }}(\rangle\right) \simeq \mathcal{C}_{\text {odd }}(\nearrow) / \mathcal{C}_{\text {odd }}(\asymp)$ (see also Figure 5). Here, $\asymp$ and ) ( depict link diagrams where a single crossing $X$ is resolved in a negative or, respectively, positive direction. This results in a short exact sequence of non-graded chain complexes:

$$
0 \longrightarrow \mathcal{C}_{\text {odd }}(\asymp) \stackrel{\text { in }}{\longrightarrow} \mathcal{C}_{\text {odd }}\left(\text { }\left(\stackrel{p}{\longrightarrow} \mathcal{C}_{\text {odd }}(\rangle\right)() \longrightarrow 0,\right.
$$

where in is the inclusion and $p$ is the projection.

In order to introduce grading into (3.1), we need to consider the cases when the crossing to be resolved is either positive or negative. We get (see Ra2):

$$
\begin{aligned}
& 0 \longrightarrow \mathcal{C}_{\text {odd }}(\asymp)\{2+3 \omega\}[1+\omega] \stackrel{i n}{\longrightarrow} \mathcal{C}_{\text {odd }}(\stackrel{\Upsilon}{\Upsilon}) \stackrel{p}{\longrightarrow} \mathcal{C}_{\text {odd }}(\supset)\{1\} \longrightarrow 0 \text {, } \\
& \left.0 \longrightarrow \mathcal{C}_{\text {odd }}()\right)\{-1\} \stackrel{i n}{\longrightarrow} \mathcal{C}_{\text {odd }}\left(\bigwedge^{\mathbb{N}} \stackrel{p}{\longrightarrow} \mathcal{C}_{\text {odd }}(\asymp)\{1+3 \omega\}[\omega] \longrightarrow 0,\right.
\end{aligned}
$$

where $\omega$ is the difference between the numbers of negative crossings in the unoriented resolution $\asymp$ (it has to be oriented somehow in order to define its Khovanov chain complex) and in the original diagram. The notation $\mathcal{C}_{\text {odd }}[k]$ is used to represent a shift in the homological grading of a complex $\mathcal{C}_{\text {odd }}$ by $k$. The graded versions of $i n$ and $p$ are both homogeneous, that is, have bidegree $(0,0)$.

By passing to homology in (3.2), we get the following result.

3.3.A. Theorem (Ozsváth-Rasmussen-Szabó ORS, see also Ra2 ). The Khovanov homology is subject to the following long exact sequences:

$$
\begin{aligned}
& \cdots \longrightarrow \mathcal{H}_{\text {odd }}(\rangle()\{1\} \stackrel{\partial}{\longrightarrow} \mathcal{H}_{\text {odd }}(\asymp)\{2+3 \omega\}[1+\omega] \stackrel{i n_{*}}{\longrightarrow} \mathcal{H}_{\text {odd }}(\underset{+}{\nearrow}) \stackrel{p_{*}}{\longrightarrow} \mathcal{H}_{\text {odd }}(\supset)\{1\} \longrightarrow \cdots \\
& \left.\left.\cdots \longrightarrow \mathcal{H}_{\text {odd }}()\right)\{-1\} \stackrel{i n_{*}}{\longrightarrow} \mathcal{H}_{\text {odd }}\left(\bigwedge^{\wedge}\right) \stackrel{p_{*}}{\longrightarrow} \mathcal{H}_{\text {odd }}(\asymp)\{1+3 \omega\}[\omega] \stackrel{\partial}{\longrightarrow} \mathcal{H}_{\text {odd }}()^{\prime}\right)\{-1\} \longrightarrow \cdots
\end{aligned}
$$

where $i n_{*}$ and $p_{*}$ are homogeneous and $\partial$ is the connecting differential and has bidegree $(1,0)$.

Remark. Long exact sequences (3.3) work equally well over any ring $R$ and for every version of Khovanov homology. This is both a blessing and a curse. On one hand, this means that all of the properties of the even Khovanov homology that are proved using these long exact sequences (and most of them are) hold automatically true for the odd Khovanov homology as well. On the other hand, this makes it very hard to find explanations to many differences between these homology theories. 


\section{Applichtions of the odD Khovanov homology}

In this section we collect some of the more prominent applications of odd Khovanov homology. We make a special effort to compare its performance to even homology. Unfortunately, there appears to be no odd version of the Rasmussen invariant Ra1. In fact, a knot might not have any rational homology in the homological grading 0 of odd Khovanov homology at all, see Figure 9. Correspondingly, odd homology cannot be used to provide bounds on the slice genus of a knot using the current technique.

4.1. Bounds on the Thurston-Bennequin number. One of the more useful application of the odd Khovanov homology is in finding upper bounds on the Thurston-Bennequin number of Legendrian links. Consider $\mathbb{R}^{3}$ equipped with the standard contact structure $d z-y d x$. A link $K \subset \mathbb{R}^{3}$ is said to be Legendrian if it is everywhere tangent to the 2-dimensional plane distribution defined as the kernel of this 1-form. Given a Legendrian link $K$, one defines its Thurston-Bennequin number, $t b(K)$, as the linking number of $K$ with its push-off $K^{\prime}$ obtained using a vector field that is tangent to the contact planes but orthogonal to the tangent vector field of $K$. Roughly speaking, $t b(K)$ measures the framing of the contact plane field around $K$. It is well-known that the TB-number can be made arbitrarily small within the same class of topological links via stabilization, but is bounded from above.

4.1.A. Definition. For a given topological link $L$, let $\overline{t b}(L)$, the $T B$-bound of $L$, be the maximal possible TB-number among all the Legendrian representatives of $L$. In other words, $\overline{t b}(L)=\max _{K}\{t b(K)\}$, where $K$ runs over all the Legendrian links in $\mathbb{R}^{3}$ that are topologically isotopic to $L$.

Finding TB-bounds for links has attracted considerable interest lately, since they can be used to demonstrate that certain contact structures on $\mathbb{R}^{3}$ are not isomorphic to the standard one. Such bounds can be obtained from the Bennequin and SliceBennequin inequalities, degrees of HOMFLYPT and Kauffman polynomials, Knot Floer homology, and so on (see [Ng1] for more details). The TB-bound coming from the Kauffman polynomial is usually one of the strongest, since most of the others incorporate another invariant of Legendrian links, the rotation number, into the inequality. In Ng1, Lenhard Ng used Khovanov homology to define a new bound on the TB-number.

4.1.B. TheOrem ( $\mathrm{Ng} \mathrm{Ng} 1)$. Let $L$ be an oriented link. Then

$$
\overline{t b}(L) \leq \min \left\{k \mid \bigoplus_{j-i=k} \mathcal{H}^{i, j}(L ; R) \neq 0\right\} .
$$

Moreover, this bound is sharp for alternating links.

This Khovanov bound on the TB-number is often better than those that were known before. There are only two prime knots with up to 13 crossings for which the Khovanov bound is worse than the one coming from the Kauffman polynomial Ng1]. There are 45 such knots with at most 15 crossings.

4.1.C. Example. Figure 8 shows computations of the Khovanov TB-bound for the $(4,-5)$-torus knot. The Khovanov homology groups in 4.1) can be used over any ring $R$, and this example shows that the bound coming from the integral homology is sometimes better than the one from the rational one, due to a strategically 


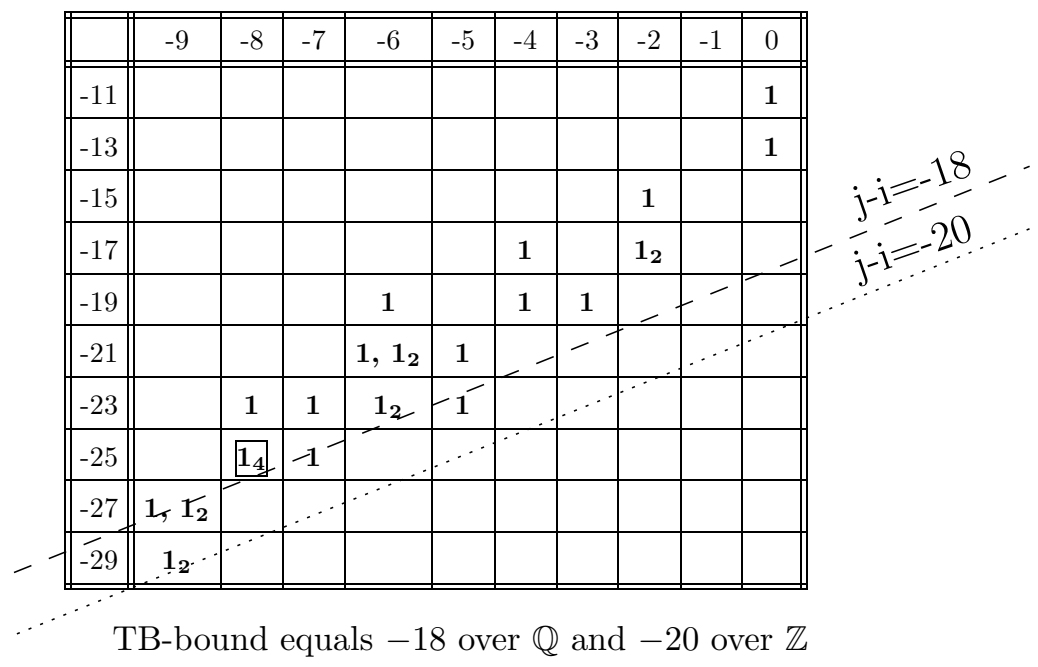

FiguRE 8. Khovanov TB-bound for the $(4,-5)$-torus knot

placed torsion. It is interesting to note that the integral Khovanov bound of -20 is computed incorrectly in Ng1. In particular, this was one of the cases where $\mathrm{Ng}$ thought that the Kauffman polynomial provides a better one. In fact, the TB-bound of -20 is sharp for this knot.

The proof of Theorem 4.1.B is based on the long exact sequences 3.3 and, hence, can be applied verbatim to the reduced as well as odd Khovanov homology. By taking into account a grading shift in the reduced homologies, we immediately get that

$$
\begin{aligned}
& \overline{t b}(L) \leq-1+\min \left\{k \mid \bigoplus_{j-i=k} \widetilde{\mathcal{H}}^{i, j}(L ; R) \neq 0\right\} \\
& \overline{t b}(L) \leq-1+\min \left\{k \mid \bigoplus_{j-i=k} \widetilde{\mathcal{H}}_{\text {odd }}^{i, j}(L ; R) \neq 0\right\} .
\end{aligned}
$$

As it turns out, the odd Khovanov TB-bound is often better than the even one. In fact, computations with KhoHo and Knotscape show that the odd Khovanov homology provides the best upper bound on the TB-number among all currently known ones for all prime knots with at most 15 crossings. More specifically, the odd Khovanov TB-bound equals the Kauffman one on all the 45 knots with at most 15 crossings where the latter is better than the even Khovanov TB-bound. These knots are $12_{475}^{n}, 13_{1708}^{n}, \overline{14}_{9580}^{n}, \overline{14}_{9989}^{n}, 14_{12208}^{n}, 14_{14433}^{n}, \overline{14}_{15458}^{n}, 14_{18373}^{n}, 14_{21980}^{n}, 14_{24190}^{n}$, $\overline{15}_{24518}^{n}, 15_{34445}^{n}, 15_{34827}^{n}, \overline{15}_{37632}^{n}, 15_{40088}^{n}, \overline{15}_{40854}^{n}, \overline{15}_{42851}^{n}, \overline{15}_{49772}^{n}, \overline{15}_{51188}^{n}, 15_{51379}^{n}$, $15_{52894}^{n}, 15_{52993}^{n}, 15_{53039}^{n}, 15_{53226}^{n}, 15_{54333}^{n}, 15_{54502}^{n}, 15_{57673}^{n}, \overline{15}_{59594}^{n}, 15_{62334}^{n}, 15_{64226}^{n}$, $15_{72658}^{n}, \overline{15}_{76240}^{n}, 15_{79161}^{n}, 15_{85661}^{n}, \overline{15}_{92272}^{n}, \overline{15}_{100242}^{n}, \overline{15}_{101483}^{n}, 15_{103773}^{n}, 15_{124839}^{n}$, $15_{124916}^{n}, 15_{132200}^{n}, 15_{140988}^{n}, 15_{144125}^{n}, 15_{145208}^{n}$, and $15_{167608}^{n}$. On the other hand, there are many knots (several thousands) for which the Khovanov TB-bound (both even and odd) is better than the Kauffman one. The first such knot is $11_{20}^{n}$.

4.1.D. Example. The odd Khovanov TB-bound is better than the even one and is equal to the Kauffman one for the knot $12_{475}^{n}$, as shown in Figure 9. 


\begin{tabular}{|r||l|l|l|l|l|l|l|l||}
\hline \hline & 0 & 1 & 2 & 3 & 4 & 5 & 6 & 7 \\
\hline \hline 13 & & & & & & & & $\mathbf{1}$ \\
\hline 11 & & & & & & & & $\mathbf{1}_{\mathbf{2}}$ \\
\hline 9 & & & & & & $\mathbf{1}$ & $\mathbf{1}$ & \\
\hline 7 & & & & & $\mathbf{1}$ & $\mathbf{1}_{\mathbf{2}}$ & & \\
\hline 5 & & & & & $\mathbf{1}, \mathbf{1}_{\mathbf{2}}$ & & & \\
\hline 3 & & & $\mathbf{1}$ & $\mathbf{1}$ & & & & \\
\hline 1 & $\mathbf{1}$ & & $\mathbf{1}_{\mathbf{2}}$ & & & & & \\
\hline-1 & $\mathbf{1}$ & $\mathbf{1}$ & & & & & & \\
\hline \hline
\end{tabular}

(even) Khovanov homology

TB-bound equals -2

\begin{tabular}{||r||r|r|r|r|r|r|r|r||}
\hline \hline & 0 & 1 & 2 & 3 & 4 & 5 & 6 & 7 \\
\hline \hline 12 & & & & & & & & $\mathbf{1}$ \\
\hline 10 & & & & & & & $\mathbf{1}$ & \\
\hline 8 & & & & & & $\mathbf{1}$ & & \\
\hline 6 & & & & & $\mathbf{2}$ & & & \\
\hline 4 & & & & $\mathbf{1}$ & & & & \\
\hline 2 & & & $\mathbf{1}, \underline{\mathbf{1}_{3}}$ & & & & & \\
\hline 0 & & $\mathbf{1}_{\mathbf{8}}$ & & & & & & \\
\hline-2 & $\mathbf{1}_{\mathbf{3}}$ & & & & & & & \\
\hline \hline
\end{tabular}

odd reduced Khovanov homology

TB-bound equals -3

FIgURE 9. Khovanov TB-bounds for the knot $12_{475}^{n}$

4.2. Finding quasi-alternating knots. Quasi-alternating links were introduced by Ozsváth and Szabó in OS2 as a way to generalize the class of alternating links.

4.2.A. Definition. The class $\mathcal{Q}$ of quasi-alternating links is the smallest set of links such that

- the unknot belongs to $\mathcal{Q}$;

- if a link $L$ has a planar diagram $D$ such that the two resolutions of this diagram at one crossing represent two links, $L_{0}$ and $L_{1}$, with the properties that $L_{0}, L_{1} \in \mathcal{Q}$ and $\operatorname{det}(L)=\operatorname{det}\left(L_{0}\right)+\operatorname{det}\left(L_{1}\right)$, then $L \in \mathcal{Q}$ as well.

Remark. It is well-known that all non-split alternating links are quasi-alternating.

The main motivation for studying quasi-alternating links is the fact that the double branched covers of $S^{3}$ along such links are so-called $L$-spaces. A 3-manifold $M$ is called an $L$-space if the order of its first homology group $H_{1}$ is finite and equals the rank of the Heegaard-Floer homology of $M$ (see [S2]). Unfortunately, due to the recursive style of Definition 4.2.A it is often highly non-trivial to prove that a given link is quasi-alternating. It is equally challenging to show that it is not.

To determine that a link is not quasi-alternating, one usually employs the fact that such links have homologically thin Khovanov homology over $\mathbb{Z}$ and Knot Floer homology over $\mathbb{Z}_{2}$ (see $\underline{\mathrm{MO}}$ ). Thus, $\mathbb{Z} \mathrm{H}$-thick knots are not quasi-alternating. There are 12 such knots with up to 10 crossings. Most of the others can be shown to be quasi-alternating by various constructions. After the work of Champanerkar and Kofman [ChK], there were only two knots left, $9_{46}$ and $10_{140}$, for which it was not known whether they are quasi-alternating or not. Both of them have homologically thin Khovanov and Knot Floer homology.

As it turns out, odd Khovanov homology is much better at detecting quasialternating knots. The proof of the fact that such knots are $\mathbb{Z H}$-thin is based on the long exact sequences (3.3) and, therefore, can be applied verbatim to the odd homology as well ORS. Computations with KhoHo show that the knots $9_{46}$ and $10_{140}$ have homologically thick odd Khovanov homology and, hence, are not quasi-alternating, see Figures 10 and 11 . 


\begin{tabular}{|r||l|l|l|l|l|l|l||}
\hline \hline & -6 & -5 & -4 & -3 & -2 & -1 & 0 \\
\hline \hline 0 & & & & & & & $\mathbf{2}$ \\
\hline-2 & & & & & & $\mathbf{1}$ & \\
\hline-4 & & & & & $\mathbf{1}$ & & \\
\hline-6 & & & & $\mathbf{2}$ & & & \\
\hline-8 & & & $\mathbf{1}$ & & & & \\
\hline-10 & & $\mathbf{1}$ & & & & & \\
\hline-12 & $\mathbf{1}$ & & & & & & \\
\hline \hline
\end{tabular}

(even) reduced Khovanov homology

\begin{tabular}{||r||c|c|c|c|c|c|c||}
\hline \hline & -6 & -5 & -4 & -3 & -2 & -1 & 0 \\
\hline \hline 0 & & & & & & & $\mathbf{2}$ \\
\hline-2 & & & & & & $\mathbf{1}$ & $\mathbf{\mathbf { 1 } _ { 3 }}$ \\
\hline-4 & & & & & $\mathbf{1}$ & & \\
\hline-6 & & & & $\mathbf{2}$ & & & \\
\hline-8 & & & $\mathbf{1}$ & & & & \\
\hline-10 & & $\mathbf{1}$ & & & & & \\
\hline-12 & $\mathbf{1}$ & & & & & & \\
\hline \hline
\end{tabular}

odd reduced Khovanov homology

Figure 10. Khovanov homology of $9_{46}$, the $(3,3,-3)$-pretzel knot

\begin{tabular}{|r||l|l|l|l|l|l|l|l||}
\hline \hline & -7 & -6 & -5 & -4 & -3 & -2 & -1 & 0 \\
\hline \hline 0 & & & & & & & & $\mathbf{1}$ \\
\hline-2 & & & & & & & $\mathbf{1}$ & \\
\hline-4 & & & & & & $\mathbf{1}$ & & \\
\hline-6 & & & & & $\mathbf{1}$ & & & \\
\hline-8 & & & & $\mathbf{2}$ & & & & \\
\hline-10 & & & $\mathbf{1}$ & & & & & \\
\hline-12 & & $\mathbf{1}$ & & & & & & \\
\hline-14 & $\mathbf{1}$ & & & & & & & \\
\hline \hline
\end{tabular}

(even) reduced Khovanov homology

\begin{tabular}{||r||r|l|l|l|l|l|l|l||}
\hline \hline & -7 & -6 & -5 & -4 & -3 & -2 & -1 & 0 \\
\hline \hline 0 & & & & & & & & $\mathbf{1}$ \\
\hline-2 & & & & & & & $\mathbf{1}$ & \\
\hline-4 & & & & & & $\mathbf{1}$ & $\mathbf{\mathbf { 1 } _ { 3 }}$ & \\
\hline-6 & & & & & $\mathbf{1}$ & & & \\
\hline-8 & & & & $\mathbf{2}$ & & & & \\
\hline-10 & & & $\mathbf{1}$ & & & & & \\
\hline-12 & & $\mathbf{1}$ & & & & & & \\
\hline-14 & $\mathbf{1}$ & & & & & & & \\
\hline \hline
\end{tabular}

odd reduced Khovanov homology

FiguRE 11. Khovanov homology of $10_{140}$, the $(3,4,-3)$-pretzel knot

Remark. It is worth mentioning that the knots $9_{46}$ and $10_{140}$ are $(3,3,-3)$ - and $(3,4,-3)$-pretzel knots, respectively (see Figure 12 for the definition). Computations show that $(n, n,-n)$ - and $(n, n+1,-n)$-pretzel links for $n \leq 6$ all have torsion of order $n$ outside of the main diagonal that supports the free part of the homology. This suggest a certain $n$-fold symmetry on the odd Khovanov chain complexes for these pretzel links that cannot be explained by the construction.

Remark. Joshua Greene has recently identified Gr all quasi-alternating pretzel links by considering 4-manifolds that are bounded by the double branched covers of the links. In particular, he found several knots that are not quasi-alternating, yet are both $\mathrm{H}$-thin and $\mathrm{OH}$-thin. The smallest such knot is $11_{50}^{n}$.

4.3. Transversely non-simple knots. Consider once again $\mathbb{R}^{3}$ equipped with the standard contact structure $d z-y d x$. A knot $K \subset \mathbb{R}^{3}$ is said to be transverse if it is everywhere transverse to the contact planes (cf. Section 4.1). Given a transverse knot $K$, one defines its self-linking number, sl $(K)$, as the linking number of $K$ with its push-off $K^{\prime}$ along the vector field $v=\frac{\partial}{\partial y}$. This vector field always lies in the contact planes. 

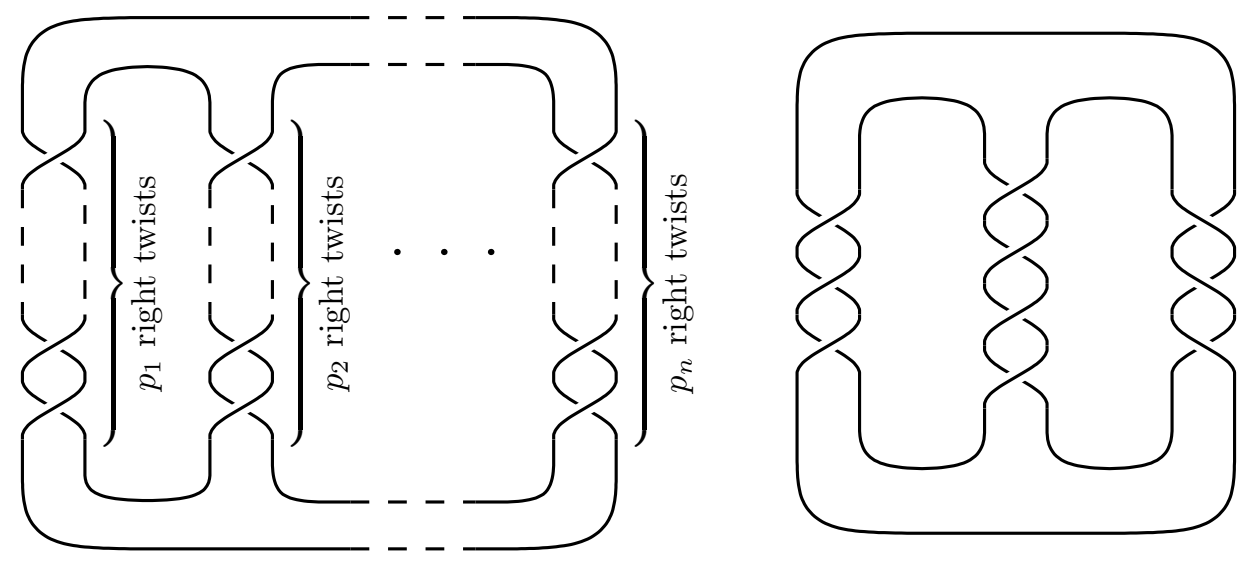

FiguRE 12. $\left(p_{1}, p_{2}, \ldots, p_{n}\right)$-pretzel link and $(3,4,-3)$-pretzel knot $10_{140}$

4.3.A. Definition. A topological knot $K$ is said to be transversely non-simple, if it has two transverse representatives $K_{1}$ and $K_{2}$ such that $\operatorname{sl}\left(K_{1}\right)=\operatorname{sl}\left(K_{2}\right)$, but $K_{1} \neq K_{2}$ as transverse knots.

The first examples of transversely non-simple knots were discovered in 2003. Birman and Menasco found a family of transversely non-simple 3-braids [BM1, BM2], while Etnyre and Honda proved that the $(2,3)$-cable of the trefoil is transversely non-simple as well [EH]. More recently, several families of transversely non-simple knots were found using knot Floer homology [B, NOT, V].

It turns out that many of these transversely non-simple knots have very special odd Khovanov homology. Namely, they have only torsion and no rational homology in the homological grading 0 . We call such knots zero-omitting. There are 677 zeroomitting knots among all prime knots with at most 15 crossings. 10 of them have 12 crossings or less: $10_{132}, 11_{38}^{n}, 12_{120}^{n}, 12_{199}^{n}, 12_{200}^{n}, 12_{260}^{n}, 12_{475}^{n}, 12_{523}^{n}, 12_{549}^{n}$, and $12_{673}^{n}$. Homology of one of these knots is shown in Figure 9. Seven out of 10 of the zero-omitting knots with at most 12 crossings are known to be transversely non-simple $\mathrm{Ng} 2$. The three unknown cases are $11_{38}^{n}, 12_{475}^{n}$, and $12_{673}^{n}$. On the other hand, the $(2,3)$-cable of the trefoil, a transversely non-simple knot, is not zero-omitting.

4.3.B. Conjecture. All zero-omitting knots are transversely non-simple.

\section{REFERENCES}

[B] J. Baldwin, Comultiplication in link Floer homology and transversely nonsimple links, Algebr. Geom. Topol. 10 (2010), no. 3, 1417-1436; arXiv:0910.1102

[BN1] D. Bar-Natan, On Khovanov's categorification of the Jones polynomial, Alg. Geom. Top., 2 (2002), 337-370; arXiv:math.QA/0201043

[BM1] J. Birman, W. Menasco, Stabilization in the braid groups. II. Transversal simplicity of knots, Geom. Topol. 10 (2006), 1425-1452 (electronic); arXiv:math/0310280

[BM2] J. Birman, W. Menasco, A note on closed 3-braids, Commun. Contemp. Math. 10 (2008), suppl. 1, 1033-1047; arXiv:0802.1072.

[B] J. Bloom, Odd Khovanov homology is mutation invariant, Math. Res. Lett. 17 (2010), no. 1, 1-10; arXiv:0903.3746.

[ChK] A. Champanerkar and I. Kofman, Twisting quasi-alternating links, Proc. Amer. Math. Soc. 137 (2009), 2451-2458; arXiv:0712.2590 
[DGShT] N. Dunfield, S. Garoufalidis, A. Shumakovitch, and M. Thistlethwaite, Behavior of knot invariants under genus 2 mutation, New York J. Math. 16 (2010), 99-123; arXiv:math.GT/0607258

[EH] J. Etnyre, K. Honda, Cabling and transverse simplicity, Ann. of Math. (2) 162 (2005), no. $3,1305-1333$; arXiv:math/0306330

[Gr] J. Greene, Homologically thin, non-quasi-alternating links, Math. Res. Lett. 17 (2010), no. 1, 39-49; arXiv:0906.2222

[HTh] J. Hoste and M. Thistlethwaite, Knotscape - a program for studying knot theory and providing convenient access to tables of knots, http://www.math.utk.edu/ morwen/knotscape.html

[J] V. Jones, A polynomial invariant for knots via von Neumann algebras, Bull. Amer. Math. Soc. 12 (1985), 103-111.

[Kh1] M. Khovanov, A categorification of the Jones polynomial, Duke Math. J. 101 (2000), no. 3, 359-426; arXiv:math.QA/9908171.

[Kh2] M. Khovanov, Patterns in knot cohomology I, Experiment. Math. 12 (2003), no. 3, 365-374; arXiv:math.QA/0201306

[L] E. S. Lee, An endomorphism of the Khovanov invariant, Adv. Math. 197 (2005), no. 2, 554-586; arXiv:math.GT/0210213

[MO] C. Manolescu, P. Ozsváth, On the Khovanov and knot Floer homologies of quasi-alternating links in Proc. of Gökova Geometry-Topology Conference 2007, 60-81, Gökova Geometry/Topology Conference (GGT), Gökova, 2008; arXiv:0708.3249.

[Ng1] L. Ng, A Legendrian Thurston-Bennequin bound from Khovanov homology, Algebr. Geom. Topol. 5 (2005) 1637-1653; arXiv:math.GT/0508649.

[Ng2] L. Ng, private communications.

[NOT] L. Ng, P. Ozsváth, D. Thurston, Transverse Knots Distinguished by Knot Floer Homology, J. Sympl. Geom. 6 (2008), no. 4, 461-490; arXiv:math/0703446

[ORS] P. Ozsváth, J. Rasmussen, and Z. Szabó, Odd Khovanov homology, Alg. Geom. Top. 13 (2013), 1465-1488; arXiv:0710.4300

[OS1] P. Ozsváth and Z. Szabó, Holomorphic disks and topological invariants for closed three-manifolds, Ann. of Math. 159 (2004) 1027-1158; arXiv:math.SG/0101206

[OS2] P. Ozsváth and Z. Szabó, On the Heegaard Floer homology of branched double-covers, Adv. Math. 194 (2005), no. 1, 1-33; arXiv:math.GT/0309170

[Ra1] J. Rasmussen, Khovanov homology and the slice genus, Invent. Math. 182 (2010), no. 2, 419-447; arXiv:math.GT/0402131

[Ra2] J. Rasmussen, Knot polynomials and knot homologies, Geometry and Topology of Manifolds (Boden et al eds.), Fields Institute Communications 47 (2005) 261-280, AMS; arXiv:math.GT/0504045

[Ro] D. Rolfsen, Knots and Links, Publish or Perish, Mathematics Lecture Series 7, Wilmington 1976.

[Sh1] A. Shumakovitch, KhoHo - a program for computing and studying Khovanov homology, https://github.com/AShumakovitch/KhoHo

[Sh2] A. Shumakovitch, Torsion of the Khovanov homology, Fund. Math. 225 (2014), 343-364; arXiv:math.GT/0405474

[V] V. Vértesi, Transversely nonsimple knots, Algebr. Geom. Topol. 8 (2008), no. 3 , 1481-1498; arXiv:0712.2803.

[W1] S. Wehrli, Khovanov Homology and Conway Mutation, arXiv:math/0301312

[W2] S. Wehrli, Mutation invariance of Khovanov homology over $\mathbb{F}_{2}$, Quantum Topol. 1 (2010), no. 2, 111-128; arXiv:0904.3401

Department of Mathematics, The George Washington University, Phillips Hall, 801 22ND St. NW, Suite \#739, Washington, DC 20052, U.S.A.

E-mail address: Shurik@gwu.edu 